\title{
Seguridad social y salud ocupacional en las empresas productoras de flores del departamento de Boyacá
}

\section{Social security and occupational health in flowers producer companies in Boyacá department}

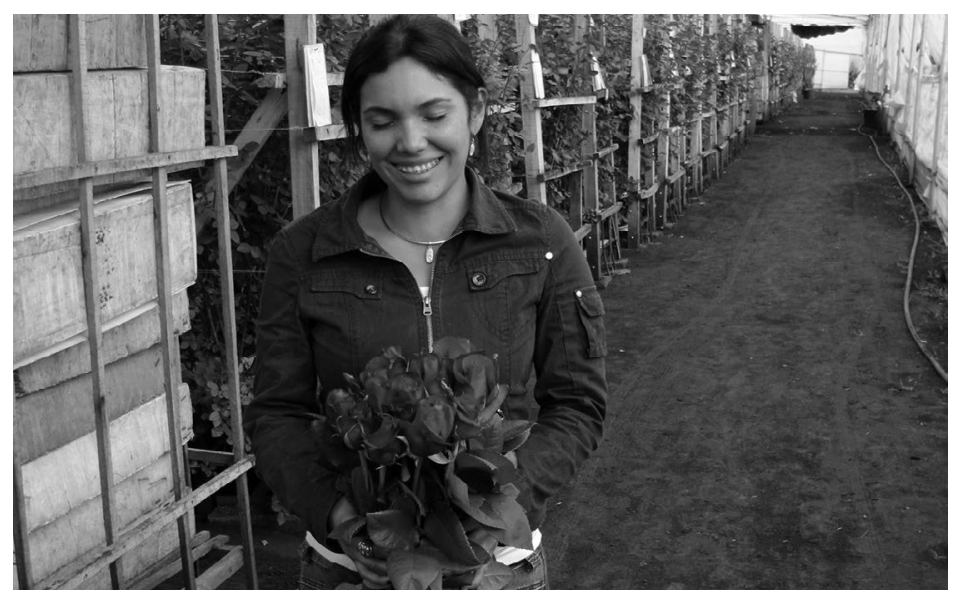

JOSÉ JAVIER GONZÁLEZ M. ${ }^{1,3}$

DIANA CAROLINA MERCHÁN F. ${ }^{1}$

LUIS FELIPE RODRÍGUEZ C. ${ }^{2}$

La floricultura colombiana, desde hace varias décadas mantiene una importante presencia en mercados nacionales e internacionales, gracias al volumen de producción y calidad de las flores.

Foto: G. Fischer

\section{RESUMEN}

La seguridad social tan tratada en estos tiempos se viene constituyendo en un factor de indiscutible importancia para el desarrollo empresarial agropecuario y, en especial, para la rama productiva de la floricultura por el uso intensivo de mano de obra, a pesar de la visión gerencial economicista en torno al tema. El presente artículo aborda la situación de la seguridad social y la salud ocupacional en empresas floricultoras del departamento de Boyacá (Colombia); en estas se realizó una caracterización y análisis de dichos sistemas. En lo concerniente a la parte metodológica, el tipo y método de estudio fue el descriptivo e inductivo; la técnica estadística utilizada fue el análisis descriptivo (frecuencias y tablas de contingencia). Como resultados relevantes se destacan, entre otros, que el $90 \%$ de la mano de obra es directa, igualmente en lo que atañe al proceso de consultoría y asesoría a las empresas floricultoras en temas de salud y pensiones, estas alcanzaron un $85 \%$, mientras que en lo referente al tema de riesgos profesionales, el nivel de asesoría e intervención alcanzó un $57,14 \%$ de apoyo efectivo. Igualmente, en cuanto a la salud se precisa que se encuentran afiliados el $85,71 \%$. En el tema de pensión el $57,14 \%$ no se encuentra afiliado al sistema. También es de resaltar que los programas de salud ocupacional si bien existen no se ponen en práctica en la mayoría de los casos.

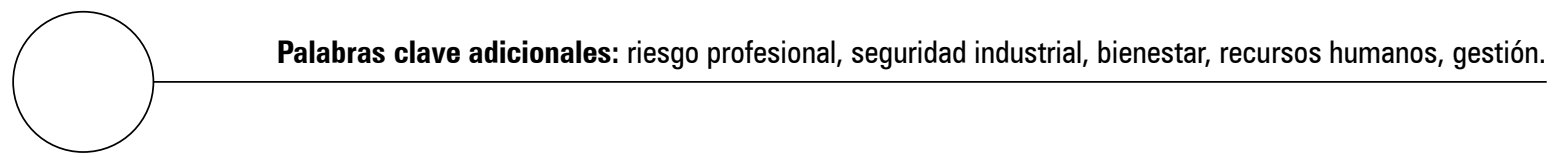

Facultad Sede Seccional Sogamoso, Escuela de Administración de Empresas, Universidad Pedagógica y Tecnológica de Colombia (UPTC), Sogamoso (Colombia).

2 Facultad de Agronomía, Departamento de Agronomía, Universidad Nacional de Colombia, Bogotá (Colombia).

3 Autor para correspondencia. javier.gonzalezmillan@uptc.edu.co 


\section{ABSTRACT}

Social security has become an indisputably important factor for agricultural business development, especially for the floriculture production branch, despite the economist management vision on this subject. This article addresses the situation of social security and occupational health in flower companies of the Boyacá department (Colombia) with a characterization and analysis of these systems. The present study used a descriptive and inductive methodology and a descriptive analysis (frequencies and contingency tables) for the statistical technique. Notably, the results showed that $90 \%$ of the manual labor is direct, also consulting and advising flower companies in health and pensions amounted to 85\%; while for occupational risks, the level of counseling and intervention reached $57.14 \%$ of the effective support. Also, $85.71 \%$ of the employees have health insurance but $57.14 \%$ are not affiliated with the pension program. Remarkably, when occupational health programs exist, they are not put into practice in most cases.

Additional key words: professional risk, safety, welfare, human resources, management.

Fecha de recepción: 07-03-2013

Aprobado para publicación: 28-05-2013

INTRODUCCIÓN

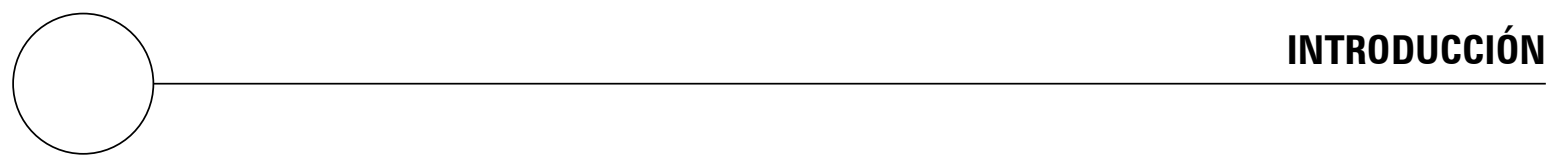

El presente artículo aborda el estudio de las características de los temas de salud, pensión, riesgos profesionales, seguridad industrial y salud ocupacional en las empresas floriculturas de Boyacá, desde la perspectiva de sus trabajadores y empresarios.

Las organizaciones de todo tipo están cada vez más preocupadas por lograr y demostrar la trascendencia de un desempeño sólido en cuanto a seguridad social y salud ocupacional (Blasina, 2007). La seguridad social, asumida con una mirada moderna, debe considerarse como medida de impacto multidimensional que beneficie a las personas y a las comunidades, deduciéndose que sin protección social adecuada será imposible tener sociedades de calidad capaces de enfrentar los retos de la globalización.

El interés por adelantar este estudio, surge de la problemática que presenta el Sistema de Seguridad Social y salud ocupacional de la rama productiva de la floricultora del departamento de
Boyacá, dada la carencia de estudios en el área de talento humano tanto en esta actividad productiva como en general en el sector agropecuario del departamento. Con el transcurrir del tiempo se ha venido evidenciando la presencia de una serie de elementos que se conjugan en el epicentro de los problemas de esta rama productiva dentro de los cuales se destacan: ausencia de un ordenamiento territorial de su expansión; crecimiento de sus problemas técnicos de producción; incidencia de variables económicas para su consolidación y posicionamiento como actividad dinámica exportadora; condiciones de vida y trabajo de los empleados florícolas; conflictos sociales y ambientales para las poblaciones aledañas, unido a su incidencia en el espacio geográfico, y el ambiente de importantes territorios (Asocolflores, 2010).

Lo anterior ha llevado a considerar la floricultura como una rama económica muy importante dentro del desarrollo social, cultural y de seguridad social dentro del departamento de Boyacá. 
No obstante, en la medida en que la actividad floricultora se expande, así mismo crecen en su contexto los conflictos ambientales, culturales, sociales y de seguridad social y ocupacional. El impacto que generan estos conflictos sobre la seguridad social, en la salud de los trabajadores, en su bienestar social y en su ambiente laboral $y$ cultural hace que exista un desequilibrio organizacional. La floricultura colombiana, desde hace varias décadas, mantiene una importante presencia en mercados nacionales e internacionales, nexo que con el proceso de globalización ha generado dentro de esta, diversos cuestionamientos y discusiones en torno al ambiente laboral, cultural, social y calidad de vida del recurso humano (Ayala, 2004).

En 1883, en Alemania, bajo el gobierno del canciller Otto Von Bismarck, aparece técnicamente la doctrina moderna de la seguridad social, que se institucionalizo en su época con la Ley del Seguro de la Enfermedad. El término "seguridad social" se vuelve común a partir de su reconocimiento en Norteamérica de la denominada "Social Security Act" en 1935. Entre los antecedentes más importantes relacionados con su consolidación se encuentra el informe de Lord Beveridge en 1942 donde se propone una reforma radical a la seguridad social y servicios asistenciales, orientada a ofrecer una protección más amplia y una cobertura universal a toda la población. Luego en 1948 se ve reforzada con la constitución del Servicio Nacional de Salud Británico (Nuget, 2000).

En Colombia, la Ley 50 de 1990, presentó en su momento la perspectiva laboral del país centrada en salario, modalidades de contrato y prestaciones, entre otras. En ese orden cronológico se da la expedición de la Constitución Política Colombiana de 1991, la que permite establecer los bien llamados derechos sociales, dentro de los cuales se consagra el tema de la seguridad social integral, la cual se regula por medio de la Ley 100 de diciembre de 1993 en la que se soporta el inicio de un nuevo y ambicioso cambio dirigido a lograr la cobertura de todos sus ciudadanos, pretendiendo transformar el viejo sistema nacional integral, de corte asistencialista estatal, caracterizado por su organización vertical y planeación centralizada, en un Sistema General de Seguridad Social en Salud, riesgos y pensión, basado en el aseguramiento, la competencia de administradoras del seguro, y prestadores de servicios, con un enorme componente de solidaridad redistributiva para financiar a los ciudadanos más desposeídos (Yépez, 2000).

Dicha norma se ve reglamentada en el tema de riesgos profesionales en 1994 por medio del Decreto 1295, el cual legisló en torno a los conceptos y formas de indemnización referentes a enfermedades profesionales, accidentes, salud ocupacional y régimen pensional del sistema (Decreto 1295, 1994). Así mismo la Ley 100 sufre su primera reforma mediante la Ley 789 de 2002, la cual buscó ampliar la protección social y apoyar el empleo (Ley 789, 2003). Luego, este mandato sufre una segunda reforma mediante la Ley 797 de 2003, en la cual se le hace una transformación importante al tema pensional presentado en la ley inicial (Ley 789).

Con respecto a la concepción del término "seguridad social" se tiene en cuenta la del español Pérez (1956) quien afirma: "la seguridad social es la parte de la ciencia política que mediante adecuadas instituciones técnicas, de ayuda, prevención o asistencia, tiene por fin defender y propulsar la paz y prosperidad general de la sociedad a través del bienestar individual de todos sus miembros". También la OIT (2003) se refiere al tema definiéndolo como "la protección que la sociedad proporciona a sus miembros, mediante una serie de medidas públicas, contra las privaciones económicas y sociales que, de no ser así, ocasionarían la desaparición o una fuerte reducción de los ingresos por causa de enfermedad, maternidad, accidente de trabajo, o enfermedad laboral, desempleo, invalidez, vejez y muerte; y también la protección en forma de asistencia médica y de ayuda a las familias con hijos". Así 
mismo, según Yepes (2000), la seguridad social se fundamenta en unos principios discriminados en básicos y operacionales así: Principios básicos: solidaridad, universalidad, integralidad, unidad e internacionalidad, y operacionales: igualdad, subsidiaridad, imprescriptibilidad, inmediación, compresividad y progresión racional.

Dentro del Sistema de Seguridad Social se contemplan varios regímenes establecidos por la Ley 100 de 1993, los que con el fin de tener claro los requerimientos de la seguridad social, presentan las siguientes características:

El régimen de seguridad social en salud está conformado por las entidades promotoras de salud y por las instituciones prestadoras de salud, a fin de posibilitar el acceso a la población afiliada a alguno de los dos regímenes; por enfermedad general y maternidad (Ley 100, 1993).

El régimen de riesgos profesionales se refiere a riesgos profesionales como el accidente que se produce como consecuencia directa del trabajo o labor desempeñada, y la enfermedad que haya sido catalogada como profesional por el Gobierno Nacional. Tiene por objeto prevenir, proteger $\mathrm{y}$ atender a los trabajadores de los efectos de las enfermedades y los accidentes que puedan ocurrirles con ocasión o consecuencia del trabajo que desarrollan, este sistema es aplicable a todos los trabajadores que laboren bajo la legislación colombiana, vinculados por contrato de trabajo de cualquier naturaleza, ya sea del sector público o privado (Ley 100, 1993).

El régimen de seguridad social en pensiones tiene por objeto amparar a la población contra las contingencias derivadas de la vejez, la invalidez y muerte por medio de pensiones y prestaciones económicas, con el objeto de aclarar un poco más la concepción de empresa, por lo que es necesario tener en cuenta los tipos de empresa según su vocación fundamental, como lo es el caso del sector agropecuario, cuya unidad productiva se denomina agroempresa la cual posee algunas características como: unidad técnico-económica en la que se realiza la producción de bienes y en donde el administrador tiene como papel la compra y combinación de insumos para transformarlos en productos para la venta.

Según Dávila (2001), las ciencias sociales y humanísticas son las que dan lugar a la realización de estudios del enfoque de las relaciones humanas, manifestada por Elton Mayo (1972) y Maslow (1970) en Estados Unidos a partir de 1923 y hacia 1933, ilustrando aspectos tan importantes como la motivación, el desarrollo personal a través del grupo, el liderazgo y el surgimiento de la concepción del homo social. Es importante destacar el papel de Von Bertalanffy, quién inicia un proceso de cambio en la concepción organizacional direccionando la caracterización de la empresa hacia un enfoque sistémico (Chiavenato, 2005).

En razón a lo anterior se hace importante contar con elementos de juicio para analizar el sistema de seguridad social presentando las distintas visiones en torno al tema. Dentro de lo pertinente se destacan las ideas de reconocidos autores iberoamericanos como Rebella (2011) en Brasil, Alazraki (2008) y Bruni (2007) en Uruguay, Winter y Obregón (2007) en Chile, y Flores (2004) y Muñoz (2008) en España, quienes coinciden en que los Sistemas Generales de Seguridad Social Integral (SGSSI) se han direccionado a la reducción de cuotas empresariales, las que se constituyen en recortes importantes en los gastos de mantenimiento del sistema, la acumulación de grandes pasivos pensionales, lo que ha conllevado a que el costo social de las reformas no esté propendiendo por el bienestar de los consumidores, sino más bien por el enriquecimiento de los grandes grupos financieros que están amparando las entidades encargadas de prestar estos servicios propios del sistema, pasando a ser de un régimen de reparto a uno de capitalización individual generando un alto riesgo como en el caso del régimen pensional en el cual la mayoría de los trabajadores tal vez no alcancen la pensión (Bucheli et al., 2006). 
Es de pensar que la visión clásica de la seguridad social ha cambiado, puesto que en otra época el trabajo era formal y fijo, lo que garantizaba el acceso al SGSSI de todo el grupo familiar, aspecto que hoy se ha modificado radicalmente fortaleciendo los contratos de corto plazo. En esta misma línea Bertranou y Sánchez (2003) refuerzan este concepto explicando el paradigma bismarkiano, el cual preceptúa como el acceso a este sistema se ve condicionado al nivel de aportes y contribuciones hechas por los empleados y trabajadores, lo que al futuro conlleva a un constante deterioro en la densidad de aportes y por ende del servicio propio de la salud, las pensiones y los riesgos profesionales.

Con respecto al tema del SGSSI para el caso colombiano, se han presentado diferentes posturas críticas, como las de Céspedes et al. (2000), quienes ponen de manifiesto que aunque la cobertura aumentó del 23 al 57\%, los indicadores de concentración y uso no lo hicieron en la misma proporción, razón preocupante para pensar que la población trabajadora efectivamente cotiza, mas no hace uso eficiente de los servicios del régimen (Restrepo, 2005).

Autores como Gaviria et al. (2006) concluyen que la cobertura del sistema evidentemente no recae en los más pobres y que en el tema de la salud, los beneficiados del régimen subsidiado no son quienes debieran ser, desaprovechando de esta manera la función de proteger a los más pobres y cayendo en la visión economicista en la cual se ha venido aumentando la cantidad mas no la calidad en los servicios originados por las reformas, esto se da por su puesto por una serie de variables como el tipo de empleo, sexo, edad, régimen de aportes, tamaño de la empresa y tipos de remuneración.

Una variable importante en este contexto la constituye el tema de la salud ocupacional definida como "las condiciones y factores que afectan o pueden afectar la salud y la seguridad de los empleados u otros trabajadores (incluidos los trabajadores temporales y personal de contrato), visitantes o cualquier otra persona en el lugar de trabajo (NTC-OHSAS 18001, 2007). Dentro de tales condiciones se destacan:

El puesto de trabajo, definido como "cualquier espacio físico en el que se realizan actividades relacionadas con el trabajo, bajo el control de la organización" (NTC-OHSAS 18001, 2007). En otras palabras es el conjunto de funciones o actividades desempeñadas por un individuo en el lugar de trabajo a cambio de una remuneración o como el trabajo total asignado a un trabajador individual, constituido por un conjunto específico de deberes y responsabilidades (Häcker, 1986).

El riesgo que se refiere a "la combinación de la probabilidad de que ocurra un(os) evento(s) o exposición(es) peligros(s), y la severidad de la lesión o enfermedad que puede ser causada por el (los) riesgo(s) o exposición(es)" (NTC-OHSAS 18001, 2007).

La enfermedad profesional es todo estado patológico que conlleva a una condición física o mental adversa que sobrevenga de alguna actividad laboral y que obviamente sea catalogada por el gobierno como tal (Decreto 1295, 1994).

Programas de salud ocupacional, dentro de estos se encuentran incluidos los elementos de protección personal, programas de salud ocupacional, reglamentos de higiene y seguridad industrial, planes de incentivos, programas de bienestar social y beneficios familiares (Novoa, 2004).

El objetivo general de esta investigación es realizar la caracterización y análisis situacional del sistema de seguridad social y salud ocupacional de las empresas floricultoras del departamento de Boyacá, con el propósito de contar con las bases para diseñar un plan de acción basado en estrategias y actividades para mejorar las condiciones laborales, culturales y sociales de la población trabajadora directamente involucrada en esta rama productiva. 


\section{METODOLOGÍA DE INVESTIGACIÓN}

Esta investigación se llevó a cabo en el departamento de Boyacá situado en el centro del país, en la cordillera oriental de los Andes. La economía de Boyacá depende de las actividades agropecuarias, de los servicios, la industria y la minería. Es aún un departamento netamente agrícola y ganadero. Sus principales cultivos son la papa (segundo productor a nivel nacional), la caña panelera, el plátano, el maíz, la yuca, la cebada y cebolla junca (primer productor a nivel nacional), y en la actualidad viene tomando una gran importancia la rama productiva de la floricultura, cuyas empresas se encuentran ubicadas en los municipios de Villa de Leyva, Duitama, Toca, Ventaquemada, Pesca, Paipa, Aquitania y Cuítiva.

Las fuentes de información primaria estuvieron constituidas por los trabajadores y empleadores de las empresas floricultoras del departamento de Boyacá. Para este tipo de fuente se utilizó como técnicas fundamentales de recolección la observación no participante o simple, apoyada en encuestas y entrevistas realizadas de forma directa en el sitio de estudio en el primer semestre de 2012.

La información secundaria se obtuvo de las bases de datos de los diferentes fondos de pensiones (Colfondos, Porvenir, Santander, Coomeva y I.S.S.) ARP (I.S.S. Salud Coop, Suratep) y EPS (I.S.S. Coomeva Salud Coop) del departamento de Boyacá y de instituciones como la Secretaría de Salud del departamento y Ministerio de Protección Social.

La población total de empresas floricultoras existentes en el departamento de Boyacá es de 11, pero para la realización de este estudio solo se tomaron siete. Para lograr una mayor confiabilidad en el estudio, en lo referente a los trabajadores, se precisó inicialmente el número total de trabajadores de la rama productiva de la floricultura del departamento de Boyacá, el cual es de 544, de donde se tomó una muestra de 126 trabajadores que corresponde al 23,1\% de la población trabajadora. El resultado de muestreo empleado fue el estratificado por afiliación proporcional, utilizando como variable de estratificación la edad del trabajador. Como herramienta fundamental para el modelamiento estadístico se utilizó el paquete STATS a través de análisis descriptivos entre variables, así mismo se utilizó el MS Excel.

\section{ANÁLISIS Y DISCUSIÓN}

En lo que respecta a la edad de la población trabajadora en las empresas floricultoras del departamento de Boyacá, el 39\% está en las edades entre los 26-30 años, un 29\%, corresponde a trabajadores entre los 31-35 años de edad y un $20 \%$ son trabajadores entre 20 y 25 años, para el intervalo de edad entre 41 a 45 años labora aproximadamente el $1 \%$ de mano de obra empleada en esas empresas (figura 1).

Con relación a la distribución de mano de obra por áreas de trabajo, el 22,22\% de los trabajadores están ubicados en el área de cosecha, un 19\% en las áreas de poscosecha y producción cada una. El área de empaque emplea el 14,00\% de la mano de obra y el 13,49\% abarca los operarios de cultivo o mano de obra que apoya a todos los anteriores. Es de destacar que el área administrativa ocupa el 5,56\% de la fuerza laboral (figura 2).

Como lo expresan importantes estudiosos del tema (Blasina, 2007; Flores, 2004), el sistema general de seguridad social siempre ha tenido graves falencias, no solo en este sector, sino en casi todos los sectores productivos; estas van desde descuidar a los jubilados, hasta el tema mismo de la falta de regulación de las cuotas empresariales para cubrir las necesidades propias del sistema. Es importante destacar que para este caso, del total de trabajadores vinculados el $90 \%$ aproximadamente es mano de obra directa calificada, es decir, personal que se encuentra en las 


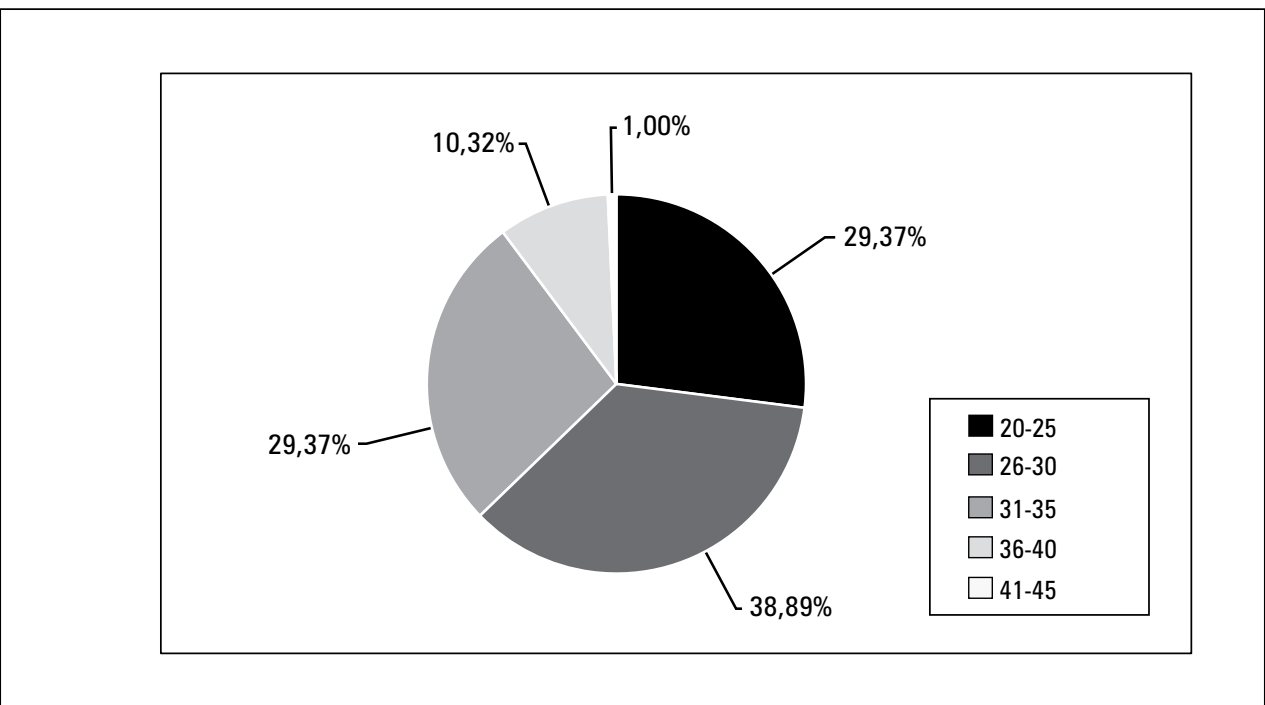

Figura 1. Edad de la población trabajadora en las empresas floricultoras del departamento de Boyacá, 2012.

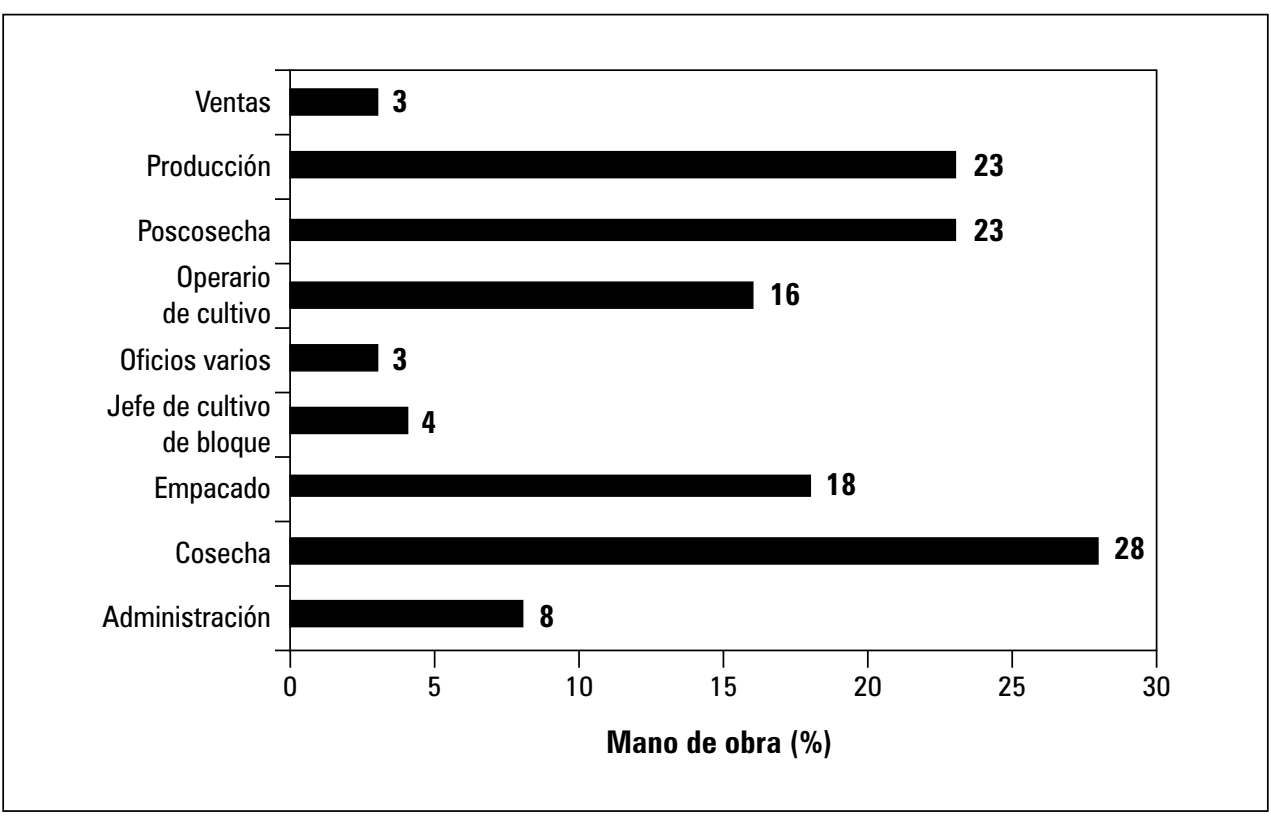

Figura 2. Distribución de mano de obra por áreas de trabajo en las empresas floricultoras de departamento de Boyacá, 2012.

tres áreas de la empresa el cual ha sido capacitado para que tengan un buen desempeño en sus labores. Tan solo un $10 \%$ es mano de obra indirecta, de la cual el $7 \%$ corresponde a mano de obra calificada que de una u otra manera tiene vinculación temporal o estacional en diferentes actividades de la empresa.
En lo concerniente a la afiliación al sistema de salud, el 95,24\% de los trabajadores de las empresas floricultoras del departamento de Boyacá, se encuentran afiliados al sistema de seguridad social y presentan la siguiente caracterización: con relación al sistema de riesgos profesionales, el $52,38 \%$ se encuentra afiliado a 
la ARP del ISS y el $32,54 \%$ a administradoras de riesgos profesionales privadas. En este contexto es preocupante que el 15,08\% de la población trabajadora no se encuentra afiliada, de lo cual deriva la total desprotección en lo que hace referencia a accidentes de trabajo y enfermedad profesional. En cuanto a salud, el 95,24\% de la población se encuentra afiliada a EPS privadas entre las que se encuentran Saludcoop, Coomeva y a las IPS, Humanavivir, Condor y Comparta. No se encuentra afiliado al régimen de salud el $4,76 \%$ de la población. En pensiones, el $85,71 \%$ se encuentran afiliados a un fondo pensional privado como Horizonte y Porvenir, $y$ el $14,29 \%$ de los trabajadores no presentan afiliación a algún régimen pensional (figura 3), aunque el problema real no radica solamente en el número de afiliados, el mayor óbice a nivel de la nación tal como opinan Salcedo (2005) y Corvera (2008), existe un rezago en términos de la capacidad de los fondos para abastecer la carga pensional actual, de la misma manera en torno a este tema, es la edad otro factor de alta influencia, que como lo ponen de manifiesto García y Moreno (2011), cada vez más los fon- dos de pensiones se optimizan, pues el esquema multifondos ha permitido incluso que sean las multinacionales las que manejen las pensiones de los trabajadores colombianos.

A pesar de que la seguridad y salud ocupacional en las organizaciones empresariales, ha sido impulsada por regulaciones gubernamentales y por la presión de sindicatos y de los mismos trabajadores, tan solo en tres empresas floricultoras del departamento de Boyacá existe el programa de salud ocupacional y en dos de ellas se desarrolla o ejecuta dicho programa, lo que permite intuir que los trabajadores están expuestos a factores de riesgo que pueden ocasionar accidentes de trabajo o enfermedades profesionales y que los patronos no son garantes de la salud de sus trabajadores, por ende de la comunidad y su entorno. De igual manera en los demás programas como seguridad industrial, medicina laboral, capacitación en salud ocupacional, prevención y desastres entre otras, tan solo el $43 \%$ de las empresas cuenta con estos programas reflejando un deficiente nivel de compromiso con los trabajadores (figura 4).

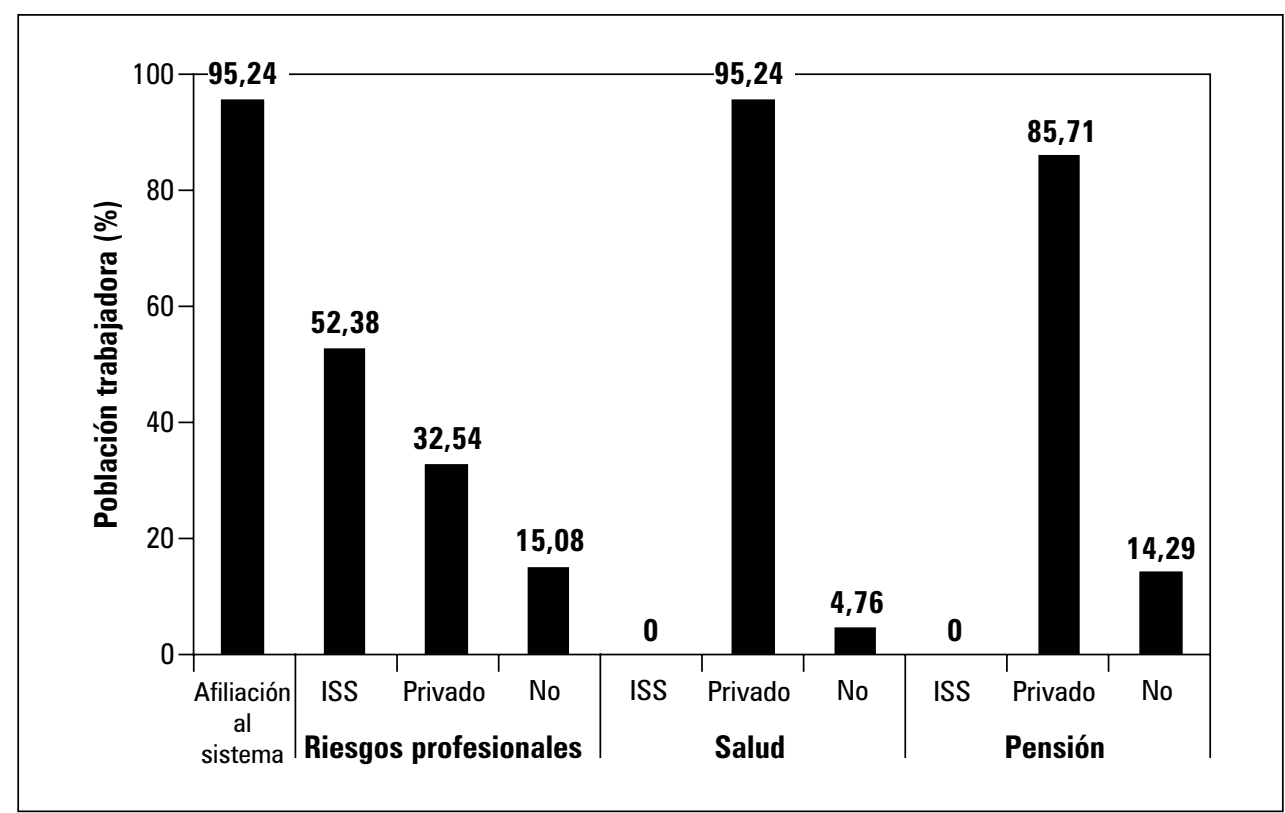

Figura 3. Distribución de la población trabajadora afiliada al sistema de seguridad social en las empresas floricultoras del departamento de Boyacá, 2012. 


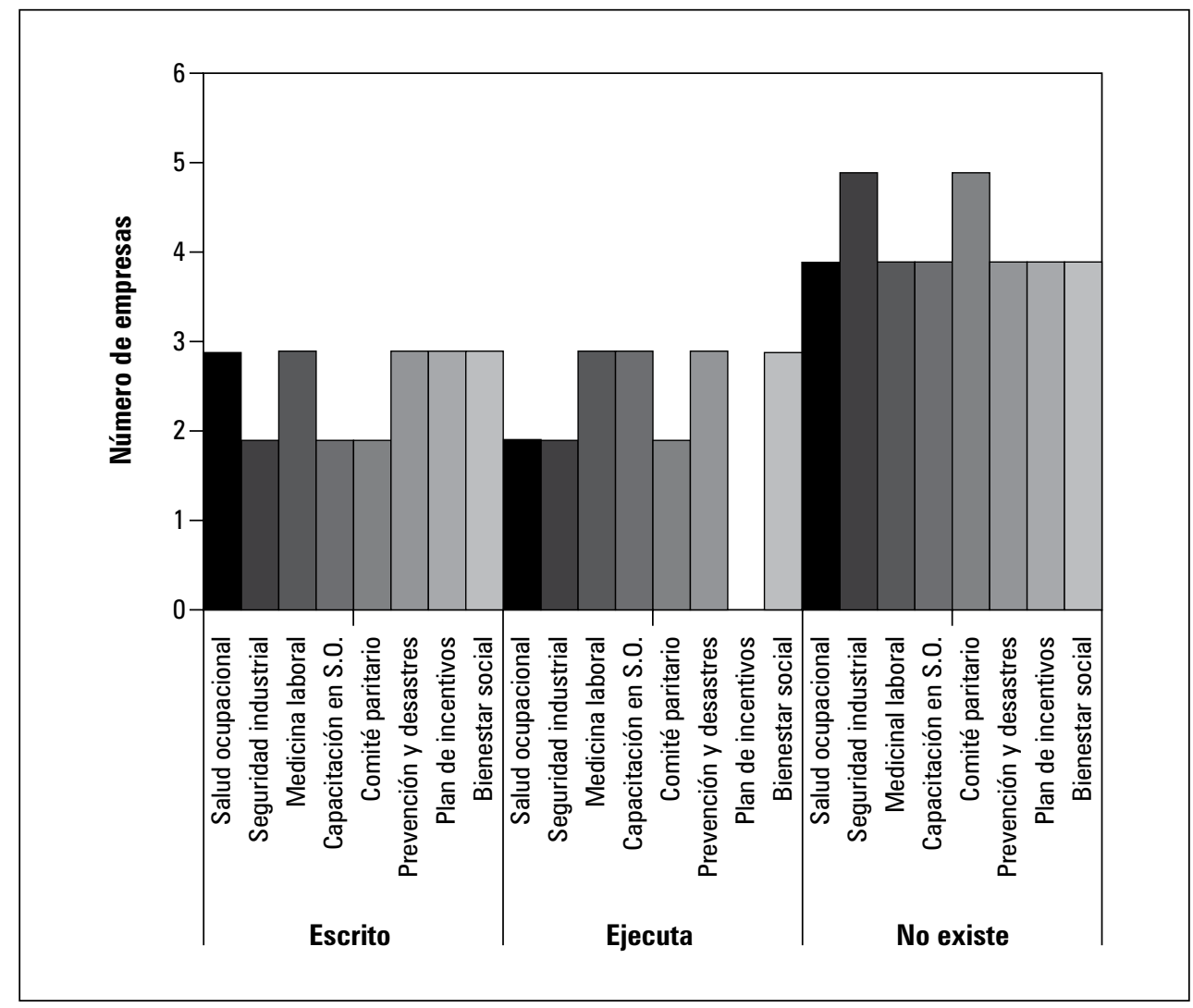

Figura 4. Existencia y ejecución de programas empresariales de seguridad y salud ocupacional de las empresas floricultoras del departamento de Boyacá, 2012.

Vale la pena destacar que tan solo en dos empresas existe el Copaso (Comité Paritario de Salud Ocupacional), como ente de vigilancia y auditoria en lo relacionado con el bienestar laboral de trabajo y salud de los trabajadores, como también es de recalcar que en tres de estas empresas existe un plan de incentivos que no se lleva a cabo.

En lo pertinente con la distribución de la afiliación al sistema de seguridad social, se hizo referencia a la afiliación de las empresas floricultoras del departamento de Boyacá al sistema de seguridad social, observándose que al régimen en salud, el 85,71\% de las empresas (Agrícola el Cactus, Flores Toca, Rokaflor, Finca el Sauz, Frutos y Flores Arco Iris y Arco Iris II) se encuentran afiliadas a E.P.S. privadas. Es notorio y muy preocupante que un sistema productivo de la importancia de las flores tenga muy pocos trabajadores afiliados a algún sistema ofrecido por parte de la seguridad social. En cuanto se refiere al régimen pensional una empresa se encuentra afiliada al ISS (ARP) y dos empresas a fondos pensionales privados; pero lo más importante y que deja una gran inquietud en cuanto al incumplimiento de la afiliación al sistema es que cuatro empresas $(57,14 \%)$ no tienen afiliados a sus trabajadores a algún régimen pensional (figura 5).

En cuanto al cumplimiento por parte del empleador de afiliación de sus trabajadores a una administradora de riesgos profesionales, solo tres empresas $(42,86 \%)$ obedecen dicho mandato $y$ se encuentran afiliadas, una al ISS (ARP) y dos a administradoras de riesgos profesionales privadas, mientras que el restante $57,14 \%$ de las empresas sus trabajadores no se encuentran afiliados a un régimen de riesgos profesionales. 


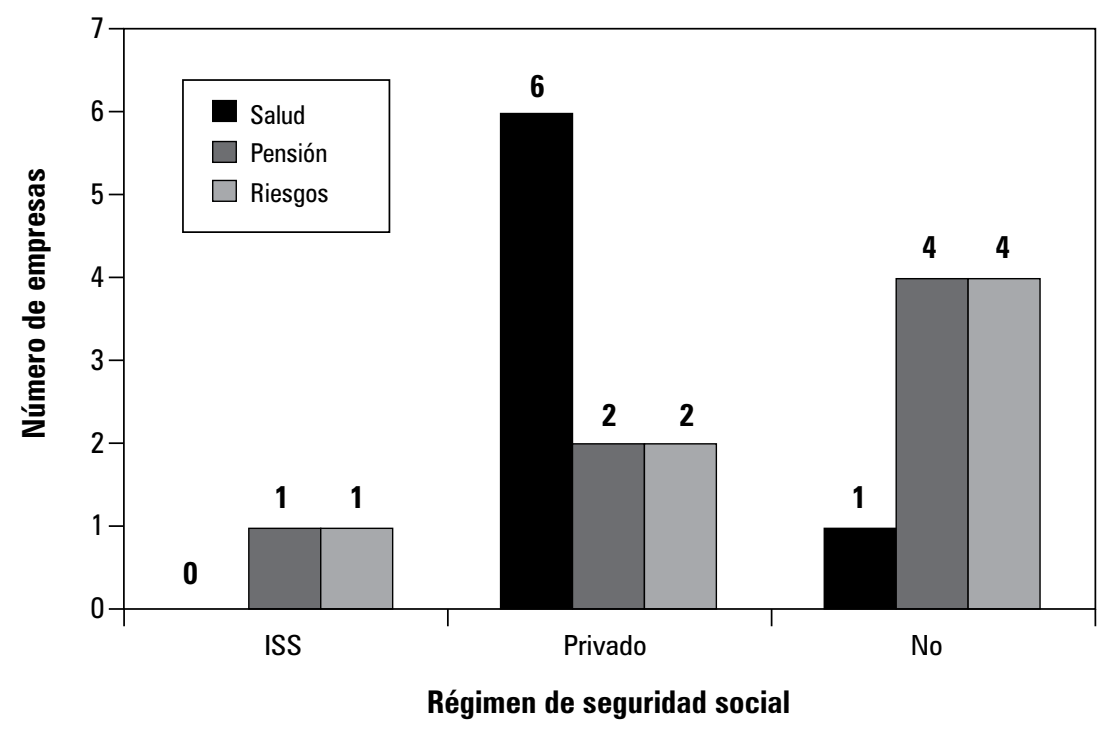

Figura 5. Distribución de afiliación a seguridad social en las empresas floricultoras del departamento de Boyacá, 2012.

En lo que respecta a la asesoría en el sistema de seguridad social por parte de las EPS, IPS, fondos pensiónales y administradoras de riesgos profesionales, se observó que se ha recibido asesoría en las empresas floricultoras en aspectos de salud y pensiones contando con el apoyo de entidades especializadas en un porcentaje mayor al $85 \%$. En lo referente a las ARP su nivel de asesoría e intervención en las empresas floricultoras de Boyacá, se puede observar que solo se ha contado con el 57,14\% de apoyo, situación argumentada en la inexistencia de sedes propias de las administradoras de riesgos profesionales en los sitios de ubicación de estas organizaciones (figura 6). Ante esta percepción autores como González (2005) y Cárdenas (2012), presentan comó el sistema pensional ha evolucionado dramáticamente, convirtiéndose en una espiral en la cual han venido aumentando los aportes y las edades; así las cosas, el bache existente en el sistema solo se remediará por medio de medidas críticas como el aumento de la edad pensional y un aporte exagerado para lograr suplir un déficit de años en el sistema.
A cerca del conocimiento de la existencia de los programas de salud ocupacional, según lo manifestado por la población trabajadora se encontró que el $76,19 \%$ conoce de tales programas. El $73,02 \%$ conoce el de seguridad industrial, ya que han trabajado anteriormente en talleres y han recibido capacitación relacionada con estos temas. Solo $35,71 \%$ de la población se han hecho participes del comité paritario de salud ocupacional (figura 7).

Por demás, se logró establecer que en cuanto al plan de incentivos solo el 46,82\% de los trabajadores conocen el programa y se han beneficiado del mismo.

En cuanto a la opinión de los trabajadores sobre la disposición y suministro de los elementos de protección personal, para el 94,44\% tales elementos son puestos a disposición en cantidad suficiente (figura 8).

La periodicidad con la cual se suministran estos elementos, está determinada de la siguiente 


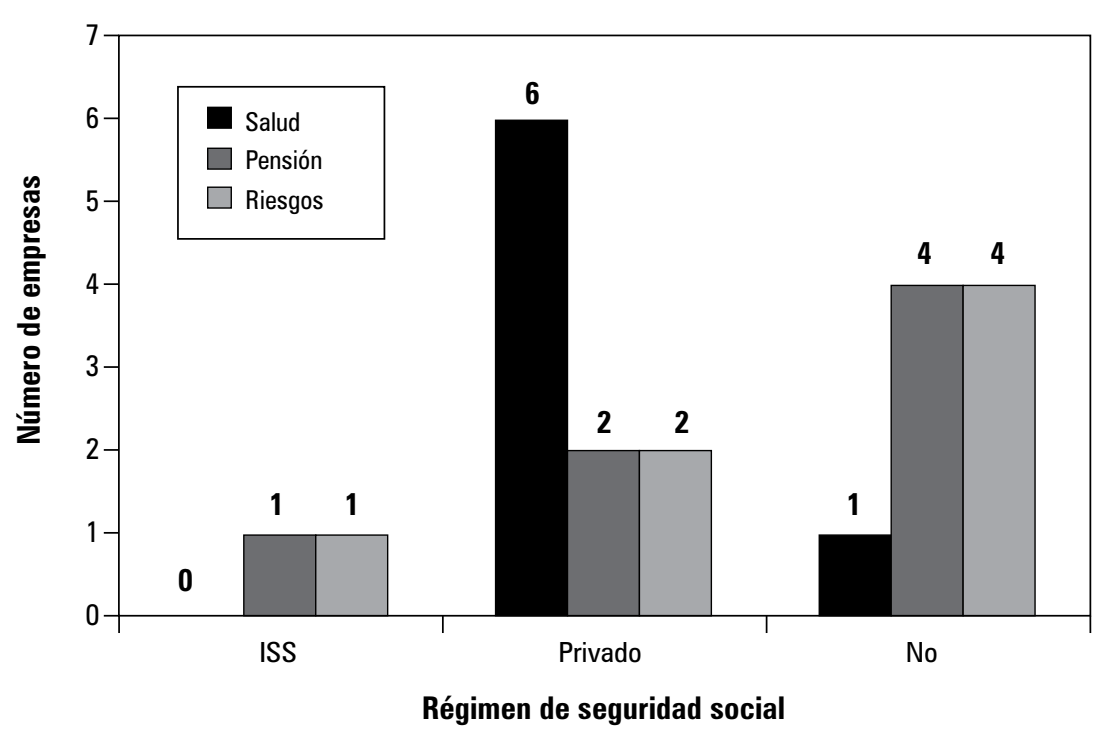

Figura 6. Asesoría recibida en el sistema de seguridad social en las empresas floricultoras del departamento de Boyacá, 2012.

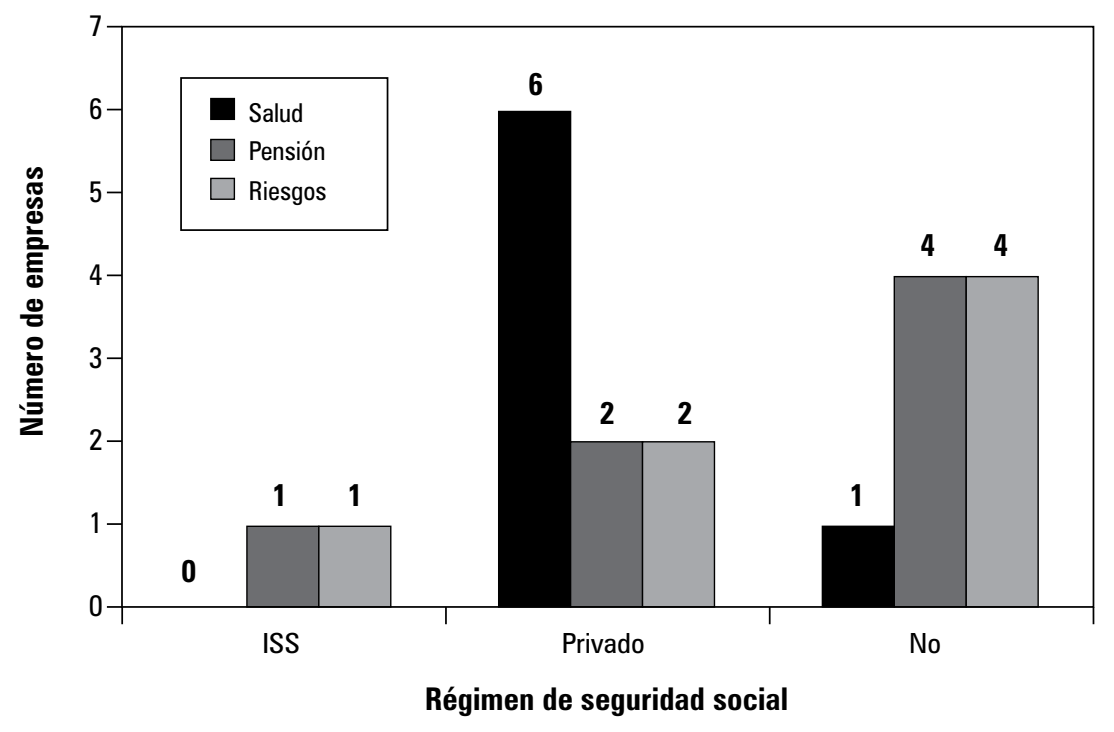

Figura 7. Porcentaje de conocimiento de los componentes del sistema de seguridad y salud ocupacional por parte de los trabajadores en las empresas de flores del departamento de Boyacá, 2012. 
manera: al 76,19\% de los trabajadores se les suministra por periodos que oscilan entre 15 y 30 d, según criterios de actividad laboral; al 16,67\% se le suministra por periodos entre 2 y 3 meses y al 7,15\% de la población trabajadora se le suministra en periodos de 4 meses. Es importante observar que según el $88,88 \%$ de los elementos suministrados son de buena calidad y permiten la protección adecuada del trabajador durante el desarrollo de sus actividades laborales (figura 8).

\section{CONCLUSIONES}

En las empresas floricultoras de Boyacá los sistemas de protección social y salud ocupacional no pueden ser vistos como caridad y costos, sino que representan una oportunidad para fortalecer el compromiso del trabajador con su empresa.

Contar con la seguridad social en salud, pensiones y riesgos profesionales es un derecho para los trabajadores de las empresas floricultoras de Boyacá y un deber para sus empleadores.

E1 95,24\% de los trabajadores de las empresas floricultoras del departamento de Boyacá se encuentran afiliados al sistema de seguridad social.

En el sistema de riesgos profesionales, el 52,38\% se encuentra afiliado a administradoras de índole público y en menor porcentaje a las ARP privadas, en tanto que el $15,08 \%$ se encuentra por fuera del sistema.

El 95,24\% de la población se encuentra afiliada a EPS privadas y estatales, en tanto que en una menor proporción $(4,76 \%)$ de la población se encuentran por fuera del sistema. En el tema pensional, el $85,71 \%$ se encuentran afiliados a un fondo, mientras que el $14,29 \%$ de los funcionarios carecen de dicha afiliación.

No se puede desconocer que en el sitio de trabajo de quienes laboran en las empresas de flores

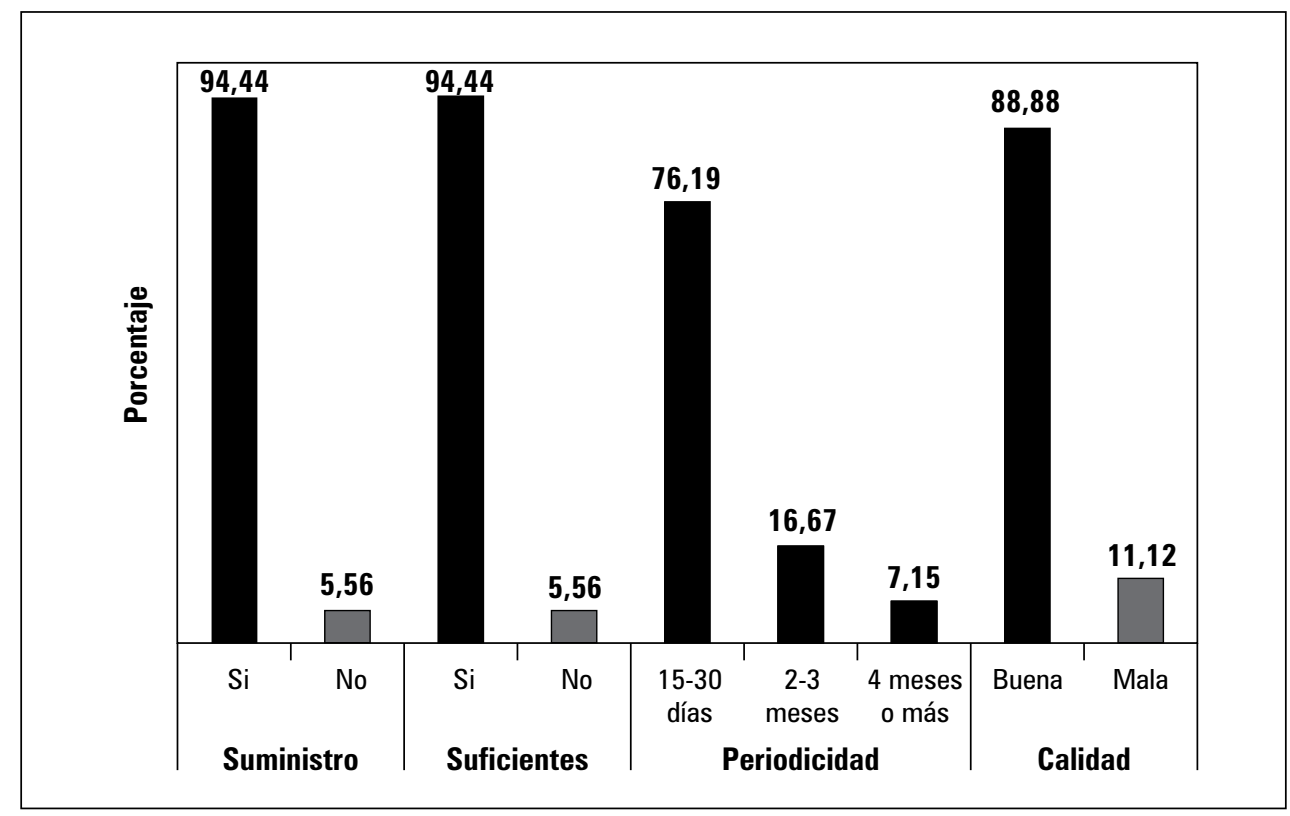

Figura 8. Valoración de la disponibilidad de los elementos de protección personal utilizados en las empresas de flores del departamento de Boyacá, 2012. 
en Boyacá, existen riesgos físicos, químicos, de seguridad, ergonómicos, psicosociales y psicológicos por lo que se hace necesaria la implementación de un riguroso sistema de gestión en seguridad y salud ocupacional.

La periodicidad con la cual se suministran los elementos de protección es relativamente corta (76,19\%), claro está que se asumió por parte de ellos que dichos elementos son los de consumo inmediato de los trabajadores (tales como guantes de látex y tapabocas), no siendo así con los elementos de calzado, vestido labor que realmente no se suministran, debido a que los contratos temporales no permiten cumplir con las tres en- tregas al año (es decir cada 4 meses); de todas formas, los elementos que en estas organizaciones se suministran son de buena calidad como lo expresa el 88,88\% de los funcionarios.

El 76,19\% del personal conoce la existencia de los programas de salud ocupacional, en tanto que el $73,02 \%$ conoce el de seguridad industrial, ya que han trabajado anteriormente en talleres y han recibido capacitación relacionada con este.

Las empresas floricultoras llevan a cabo muy poco las políticas de seguridad y salud ocupacional, pues no es suficiente con tenerlas escritas, sino ponerlas en práctica.

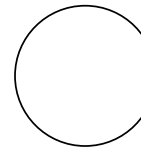

Alazraki, E. 2008. El sistema de seguridad social cuesta 2.200 millones de dólares anuales. En: La República, http://search.proquest.com/docview/466908 557 accountid=43790; consulta: febrero de 2011.

Asocolflores. 2010. Boletín informativo, Asocolflores.201; En: http://www.asocolflores.org; consulta: marzo de 2011.

Ayala, L. 2004. Cartilla dirigida al trabajador del sector floricultor. $2^{a}$ ed. Editorial Protección Laboral Seguro Social, Bogotá.

Bertranou, F. y A. Sánchez. 2003. Características y determinantes de la densidad de aportes a la seguridad social en la Argentina 1994-2001. En: Oficina Internacional del Trabajo, http://www.aaep.org. ar/espa/anales/PDF_03/Bertranou_Sanchez.pdf; consulta: mayo de 2011 .

Blasina, J. 2007. Jubilados proponen reformas al sistema de seguridad social. En: Revista Opinión, http:// search.proquest.com/docview/465659520?accoun tid $=43790$ consulta: marzo de 2011.

Bruni, J. 2007. Opinión - profundo y democrático debate requiere el sistema de seguridad social. En: Noticias Financieras, http://search.proquest.com/docvie w/465707539? accountid $=43790$; consulta: abril de 2011.

Bucheli, M., N. Ferreira-Coimbra, A. Fortesa e I. Rossi 2006. El acceso a la jubilación o pensión en Uru-

\section{REFERENCIAS BIBLIOGRÁFICAS}

guay: ว̇cuántos y quiénes lo lograrán?. En: CEPAL, http://www.eclac.cl/publicaciones/xml/4/27154/ lcw84e.pdf; consulta: febrero de 2011.

Cárdenas, R.M. 2012. La vejez sin pensiones. En: http://search.proquest.com/docview/922373968 ?accountid=43790; consulta: diciembre de 2012 .

Céspedes, J.E., I. Jaramillo, R. Martínez, S. Olaya, J. Reynales, C. Uribe, R.A. Castaño, E. Garzón, C. Almeida, C. Travassos, F. Basto y J. Angarita. 2000. Efectos de la reforma de la seguridad social en salud en Colombia sobre la equidad en el acceso y la utilización de servicios de salud. Rev. Salud Pública 2(2), 145-164.

Chiavenato, I. 2005. Introducción a la teoría general de la administración. 7ª ed. McGraw Hill, Bogotá.

Corvera, L. 2008. Situación y perspectivas del sistema privado de pensiones. Noticias Financieras. En: http://search.proquest.com/docview/466843544? accountid=43790; consulta: junio de 2012.

Dávila, C. 2001. Teorías organizacionales y administrativas: un enfoque crítico. $2^{\text {a }} \mathrm{ed}$. Mc Graw Hill, Bogotá.

Decreto 1295 de 1994. Por el cual se determina la organización y administración del Sistema General de Riesgos Profesionales. Editorial Congreso de la República, Bogotá. 
Flores, C. 2004. Evaluación de una reducción de las cuotas empresariales a la seguridad social a nivel regional a través de un modelo de equilibrio general aplicado: el caso de Andalucía. Estudios de Economía Aplicada 22(1), 99-113.

García, C.M. y J.A. Moreno. 2011. Optimización de portafolios de pensiones en Colombia: El esquema de multifondos, 2003-2010. Ecos de Economía 15(33), 139-183.

González, C. 2005. Evolución del sistema colombiano de pensiones. Portafolio. En: http://search.proquest. $\mathrm{com} /$ docview $/ 466071632$ ? accountid $=43790$; consulta: diciembre de 2012 .

Gaviria, A., C. Medina y C. Mejía. 2006. Assessing health reform in Colombia: From theory to practice/comments. Economía 7(1), 29-72.

Häcker, W. 1986. Características de la seguridad social. En: Seguridad Social. http://www.gestiopolis. com/recursos; consulta: marzo de 2011

Ley 797 de 2003. Por la cual se reforman algunas disposiciones del sistema general de pensiones previsto en la Ley 100 de 1993 y se adoptan disposiciones sobre los Regímenes Pensionales exceptuados y especiales. En: Diario Oficial del 29 de enero de 2003 del Congreso de Colombia, Bogotá.

Ley 789 de 2002. Por la cual se dictan normas para apoyar el empleo y ampliar la protección social y se modifican algunos artículos del Código Sustantivo de Trabajo. En: Diario Oficial del 27 de diciembre de 2002 del Congreso de Colombia, Bogotá.

Ley 100 de 1993. Por el cual se establece el sistema de seguridad social integral de Colombia. Editorial Congreso de la República, Bogotá.

Ley 50 de 1990. Por la cual se introducen reformas al Código Sustantivo del Trabajo y se dictan otras disposiciones. En: Diario Oficial de diciembre del 28 de 1990 del Congreso de Colombia, Bogotá.

Maslow, A. 1970. Motivation and personality. 2a ed. Harper \& Row Publisher, New York, NY.

Mayo, E. 1972. Problemas humanos de una civilización industrial. $2^{\mathrm{a}}$ ed. Editorial Nueva Visión, Buenos Aires.
Muñoz, J. 2008. The integration of the occupational safety and health in the general management business. Editorial Universidad de Deusto, Madrid.

Norma Técnica Colombiana NTC-OHSAS 18001. 2007. Sistemas de gestión en seguridad y salud ocupacional- requisitos. $2^{\mathrm{a}}$ ed. Icontec, Bogotá.

Novoa, A. 2004. Cartilla comités paritarios de salud ocupacional - Copaso. $3^{a}$ ed. Editorial Seguro Social de Boyacá - Protección Laboral, Tunja, Colombia.

Nuget, R. 2000. La seguridad social, su historia y sus Fuentes. En: Seguridad Social, http://www.bibliojuridica.org/libros/1/139/36.pdf; consulta: abril de 2011.

Organización Internacional del Trabajo (OIT). 2003. Historia de la seguridad social. En: Administración de la seguridad social, http://www.oit.org.com; consulta: noviembre de 2010

Pérez, J. 1956. Fundamentos de la seguridad social. Editorial Aguilar, Madrid.

Rebella, J. 2011. Economista y funcionario del Bondes critica el sistema de seguridad social. En: Noticias Financieras, http://search.proquest.com/docview/ 846924431 ?accountid=43790; consulta: marzo de 2011.

Restrepo, H. 2005. Health promotion today: Proposals for its future development. Global Health Promotion 12(1), 21-47.

Salcedo, E. 2005. Género, desafío de las pensiones. En: http://search.proquest.com/docview/466046138? accountid=43790; consulta: abril de 2011 .

Winter, M. y P. Obregón. 2007. Expertos analizan rol del Estado y de privados en sistema de seguridad social. En Mercurio, http://search.proquest.com/ docview/467144260? accountid =43790; consulta: abril de 2011.

Yépez, H. 2000. Legislación en salud ocupacional y riesgos profesionales. Editorial Consejo Nacional de Seguridad, Bogotá. 